\title{
Processing of Musical Syntax Tonic versus Subdominant: An Event-related Potential Study
}

\author{
Bénédicte Poulin-Charronnat ${ }^{1}$, Emmanuel Bigand ${ }^{2}$, \\ and Stefan Koelsch ${ }^{1}$
}

\begin{abstract}
The present study investigates the effect of a change in syntactic-like musical function on event-related brain potentials (ERPs). Eight-chord piano sequences were presented to musically expert and novice listeners. Instructed to watch a movie and to ignore the musical sequences, the participants had to react when a chord was played with a different instrument than the piano. Participants were not informed that the relevant manipulation was the musical function of the last chord (target) of the sequences. The target chord acted either as a syntactically stable tonic chord (i.e., a $\mathrm{C}$ major chord in the key of $\mathrm{C}$ major) or as a less syntactically stable subdominant chord (i.e., a $\mathrm{C}$
\end{abstract}

\section{INTRODUCTION}

During the last decade, the syntactic processing of musical structures has been investigated through behavioral and electrophysiological studies. A key issue was to assess whether violation of the musical syntax might affect the behavioral response on target events and which ERP components reflect the sensitivity of listeners to the violations of musical rules. The present study furthers this issue by investigating a rather subtle irregularity in the musical function of chords.

The syntactic features of Western tonal music on which some studies focused dealt with the organization of musical chords, referred to as the rules of harmony in treatises of music theory.

A chord is a simultaneous sounding of three or more tones. A chord can be built on each of the seven tones of a given key. For example, the $\mathrm{C}$ major chord is built on the tone c and is composed of the tones c, e, and $\mathrm{g}$. There are 24 major and minor chords in Western tonal music, all having a specific function depending on the key context in which they occur. That defines a so-called Western tonal hierarchy, also referred to as a hierarchy

\footnotetext{
${ }^{1}$ Max-Planck-Institute for Human Cognitive and Brain Sciences, Leipzig, Germany, ${ }^{2}$ LEAD-CNRS, Université de Bourgogne, Dijon, France
}

major chord in the key of $G$ major). The critical aspect of the results related to the impact such a manipulation had on the ERPs. An N5-like frontal negative component was found to be larger for subdominant than for tonic chords and attained significance only in musically expert listeners. These findings suggest that the subdominant chord is more difficult to integrate with the previous context than the tonic chord (as indexing by the observed N5) and that the processing of a small change in musical function occurs in an automatic way in musically expert listeners. The present results are discussed in relation to previous studies investigating harmonic violations with ERPs. of musical stability. In a given key context (say, the key of $\mathrm{C}$ major), the most stable chord is called the tonic chord (built on the first tone of the scale and noted I, the $\mathrm{C}$ major chord). All the other chords are usually perceived with reference to the tonic chord. With the dominant chord (built on the fifth tone of the scale and noted V, the G major chord) and the subdominant (built on the fourth tone of the scale and noted IV, the F major chord), the tonic chord forms the core of Western tonal harmony (Krumhansl, 1990; Bharucha \& Krumhansl, 1983). The hierarchy of stability underlies harmonic progressions. For example, at the end of musical pieces or phrases, the tonic chord often directly follows the dominant chord, creating a dominant to tonic progression denoted as authentic (or perfect) cadence and leading to the best feeling of closure. Priming studies have demonstrated that Western listeners have internalized the Western harmonic hierarchy (Bigand \& PoulinCharronnat, in press; Tillmann, Bharucha, \& Bigand, 2000 for reviews).

For example, in Bigand, Poulin, Tillmann, Madurell, and D'Adamo (2003), participants were presented with eight-chord piano sequences, with the last chord (target) either acting as a stable tonic chord, or as a less stable subdominant chord. The subdominants at the end of the sequences are very subtle harmonic irregularities, and differ, for instance, from Neapolitan chords at the end of sequences in that the subdominants 
do not represent frank expectancy violations. Results showed that participants were faster and more accurate in processing the target chord when it acted as a stable tonic chord rather than as a less stable subdominant chord. Because participants were both musicians and nonmusicians, results demonstrated that Western listeners are sensitive to the harmonic function of chords, even when they did not receive formal musical training. Behavioral studies investigating differences between musicians and nonmusicians with harmonic priming paradigms have found only few (Bigand et al., 2003) or no differences (Poulin-Charronnat, Bigand, Madurell, \& Peereman, 2005; Bigand, Tillmann, Poulin, D'Adamo, \& Madurell, 2001) between both groups of participants. These studies corroborated the hypothesis in which the syntactic structure of the Western tonal system can be implicitly acquired through passive learning processes, by mere exposure to the Western tonal music in everyday life (for a review, see Tillmann et al., 2000).

The neural mechanisms underlying these priming effects are still not well understood. Studies investigating the processing of harmonic structure with five-chord sequences using ERPs found negative components elicited by irregular chords compared to regular chords (for a review, see Koelsch \& Friederici, 2003). In these studies, harmonically irregular chord functions occurring in the middle or at the end of such sequences elicited an early right anterior negativity (ERAN), usually peaking around $200 \mathrm{msec}$ (see Patel, Gibson, Ratner, Besson, \& Holcomb, 1998 for a negative ERP component similar to the ERAN). The ERAN, which is taken to reflect the violation of a musical sound expectancy, was usually followed by a late negative frontal component that was larger for irregular than for regular (tonic) chords. This late component was denoted as the N5 and taken to reflect processes of harmonic integration (Koelsch, Schröger, \& Gunter, 2002; Koelsch, Gunter, Friederici, \& Schröger, 2000). Both ERAN and N5 invert polarity at mastoidal sites with nose reference. Moreover, both ERAN and N5 can be elicited preattentively, that is, even when the musical stimuli are ignored (Koelsch, Schröger et al., 2002), and both components can be elicited in musicians as well as nonmusicians (Koelsch, Schmidt, \& Kansok, 2002; Koelsch, Schröger et al., 2002; Koelsch et al., 2000).

Other ERP studies investigating the processing of musical syntax have reported different findings. In Regnault, Bigand, and Besson (2001), the last chord of eight-chord piano sequences acted either as a stable tonic chord or as a less stable subdominant chord, and both chord types were either consonant, or rendered dissonant. Participants had to indicate whether the last chord of each sequence was consonant or dissonant. The results indicated that changes in the harmonic function of the last chords (tonic or subdominant) modulated the amplitude of a P300 component: The P300 was larger for subdominant chords than for tonic chords, possibly reflecting top-down influences on the perceptual stages of processing (for similar effects of the harmonic function of chords on positive potentials with latencies around 300-600 msec, see also Patel et al., 1998 Janata, 1995). No negative components were found in that study.

The results reported in previous ERP studies are lacking in consensus. One way to accommodate these contradictory findings would be to consider that experimental situations differed slightly, and that these differences might have a strong influence on ERPs. Contrary to the studies of Koelsch and collaborators (2000), in Regnault et al. (2001) the participants were required to make a judgment on the target chord. The fact that the target chord was task-relevant might account for the absence of an N5 effect: The occurrence of a large P300 (perhaps related to the consonant/dissonant judgment required on the target chord) might partly overlap with the late negativity. A second difference of potential influence relates to the type of syntactic violation used. Koelsch, Schmidt, et al. (2002), Koelsch, Schröger, et al. (2002), and Koelsch et al. (2000) compared the processing of tonic and Neapolitan chords, whereas Regnault et al. (2001) compared tonic and subdominant chords. The main difference is that the Neapolitan chord does not belong to the key context while the subdominant does. As a consequence, it is likely that different types of syntactic irregularities of musical rules might be associated with different changes in the ERPs.

The present experiment was designed to address this potential concern: The same type of irregularities investigated by Regnault et al. (2001) were evaluated in an experimental design similar to the one used by Koelsch et al. (2000), in which the target chords were not taskrelevant. The eight-chord sequences of Bigand et al. (2003) were used, in which the last chord of the sequences (target) either acted as a stable tonic or as a less stable subdominant chord. In addition, to disentangle sensory and cognitive processes present in harmonic priming, the occurrence of the less stable subdominant chord in the previous context was manipulated. In one condition, neither the tonic nor the subdominant chord occurred in the previous context. In a second condition, the less stable subdominant occurred one or two times, whereas the stable tonic never occurred in the previous context. Participants were required to watch a silent movie with subtitles with instructions to detect rarely occurring piano-timbre deviants (nontarget chords that were played with a different instrumental timbre than piano, such as flute or violin).

In this preattentive experimental design, the less stable subdominant chords were expected to elicit an ERAN followed by a larger N5 compared to the stable tonic chords, with no occurrence of a P300 as target chords were not task-relevant. The amplitude of both 
the ERAN and N5 was expected to be larger for musicians than nonmusicians (Koelsch, Schmidt, et al., 2002). In addition, the manipulation of the occurrence of the less stable subdominant chord in the previous context assessed the way sensory and cognitive manipulations affect the ERPs.

Finally, as in studies from Koelsch et al. (2000), participants were explicitly required to detect timbre-deviant chords, offering the possibility of evaluating the preattentive processing of timbre deviance. The piano-timbre deviants were expected to elicit a timbre mismatch negativity (MMN) (Tervaniemi, Winkler, \& Näätänen, 1997). The MMN is elicited at 100-200 msec after the presentation of a rare deviant stimulus (i.e., timbre-deviant chords) presented among repetitive standards (i.e., piano chords). Because the MMN is elicited even when the listeners disregard auditory attention, it can be used as a preattentive index of auditory processing. The timbre MMN should be followed by a P300, as the piano-timbre deviant chords were task-relevant.

\section{METHODS}

\section{Participants}

Nineteen nonmusicians (aged 20 to 28 years; mean = 23.7 years; 10 women), and 21 musicians (aged 20 to 36 years; mean $=24.6$ years; 14 women) participated in the study.

The musicians were selected from the Conservatory for Music and Drama of Leipzig and had received, on average, 18 years of musical training (range: 11 to 29) and played an instrument (violin, piano, cello, recorder, transverse flute, contrabass, oboe, saxophone, clarinet, harpsichord, guitar, bass, trumpet, cornet). All participants were right-handed and reported to have normal hearing. They were tested individually and were paid for their participation.

\section{Stimuli}

The sequences were those used by Bigand et al. (2003). Twelve prime sequences of six chords were used. The succession of the last two chords of each sequence formed a local authentic cadence that represents a syntactic-like mark of ending in Western music (e.g., $\mathrm{G}-\mathrm{C}, \mathrm{C}-\mathrm{F}$ or $\mathrm{F}-\mathrm{B} b)$. The chord sequences thus contained eight chords, all ending on the same type of local harmonic structure (i.e., a local authentic cadence, V-I). The last chord of the sequence defined the target. The harmonic function of this target chord was manipulated so that this last chord functioned either as a stable tonic chord (I) or as a less stable subdominant chord (IV) in the key of the prime context. Figure 1 illustrates this manipulation for one of the 12 prime sequences. For each of the different 12 chord sequences, the first six chords were held constant and only the harmonic function of the last two chords was changed. The displayed prime context in Figure 1 is in the $\mathrm{C}$ major key and ends either on the chords $G$ and $C$ or on the chords $\mathrm{C}$ and $\mathrm{F}$. In the first ending, the target $\mathrm{C}$ acts as a tonic chord (I). In the second ending, the target $\mathrm{F}$ acts as a subdominant chord (IV). Crossing the 12 prime sequences with the two types of endings resulted in 24 chord sequences.

In addition to manipulating the harmonic function of the target, the occurrence of the subdominant chord in the prime context was also manipulated. In the

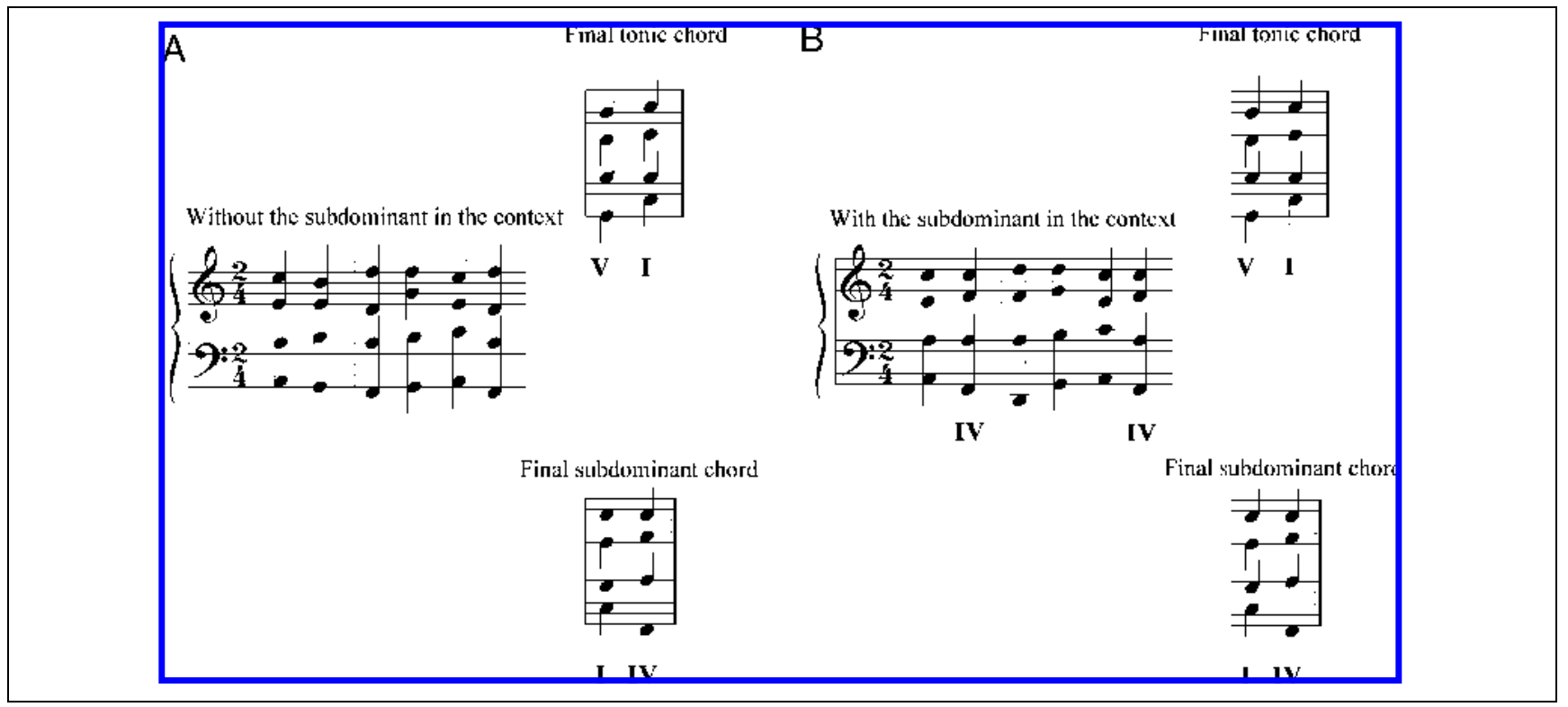

Figure 1. Examples of eight-chord sequences of Bigand et al. (2003) used in the present study. 
"no-target-in-context condition" (Figure 1A), the targets (tonic and subdominant) never occurred in the prime context. In the "subdominant-in-context condition" (Figure 1B), the less stable subdominant chord occurred once or twice in the context but the stable tonic chord never occurred in the previous context.

To increase the signal-to-noise ratio and to fit with the duration of the movie, the chord sequences were repeated resulting in 252 chord sequences in each condition. For the purpose of the timbre-deviant detection, 147 piano chord sequences were modified in order to play one chord (second, third; fourth, fifth, sixth, seventh, and eighth position) with a different timbre (flute, violin, trumpet, or harpsichord). To control for sequential effects, five different pseudorandom orders of the eight-chord sequences were used. The pseudorandom orders avoided the direct successions of sequences in the same key and the direct successions of sequences ending on the same chord function. Because of the small percentage of chords played with a different instrument (12.73\%), the sequences with a timbre-deviant chord were distributed among the standard sequences.

\section{Apparatus}

The eight-chord sequences were generated by a Macintosh computer using Cubase software (Steinberg Cubase VST Score 5.0). They were then exported in .wav format and presented to the participants at comfortable listening levels (approx. $55 \mathrm{~dB}$ ) using a pair of loudspeakers placed in front of them.

\section{Procedure}

Following the electrode application, each participant was comfortably seated in a soundproof booth, with a computer screen and two loudspeakers in front of him or her. At the beginning of the session, participants were informed that they would watch a silent movie ("You've got mail," $115 \mathrm{~min}$ ) with subtitles. The participants completed a preattentive listening task, a procedure that has already been used in previous experiments (Koelsch, Schröger, et al., 2002; Tervaniemi et al., 1997). During the movie, the eight-chord piano sequences were presented. The participants were informed that infrequently a chord was played with a different instrument than piano (flute, violin, trumpet, or harpsichord) and were asked to press a button when they detected such a deviant chord. Four examples were given to the listeners (two without and two with a deviant chord). Participants were asked to enjoy the movie and to ignore the auditory stimuli. After making sure that the participants had understood the task, both the movie and the experimental stimulus were started. There was a short break after one hour of the experiment. To avoid strong eye movements when reading the subtitles, the size of the movie window was reduced to one quarter of the original size.

\section{ERP Recording}

The electroencephalogram was recorded from 61 scalp sites of 10-20 system (Fp1, Fpz, Fp2, AF7, AF3, AFz, AF4, AF8, F9, F7, F5, F3, Fz, F4, F6, F8, F10, FT9, FT7, FC5, FC3, FCz, FC4, FC6, FT8, FT10, A1, T7, C5, C3, Cz, C4, C6, T8, A2, TP9, TP7, CP5, CP3, CPz, CP4, CP6, TP8, TP10, P9, P7, P5, P3, Pz, P4, P6, P8, P10, PO, PO3, POz, PO4, PO8, O1, Oz, O2). Horizontal eye movements were recorded with electrodes placed on the outer left and right canthus. Electrophysiological signals were digitized continuously (500-Hz sampling rate), amplified with PORTI32/MREFA (Twente Medical Systems), and stored on a hard disk for off-line analysis. The reference electrode was the left mastoid and an electrode was placed on the tip of the nose.

\section{Data Analysis}

Trials with artifacts due to eye movements and muscle activity were first eliminated by rejecting data whenever the standard deviation within a gliding window of 800 msec exceeded $50 \mu \mathrm{V}$ for the Fp1, Fpz, Fp2, AF7, $\mathrm{AF} 3, \mathrm{AFz}, \mathrm{AF} 4, \mathrm{AF} 8$, and $25 \mu \mathrm{V}$ at the remaining electrodes. The trials with artifacts were also rejected whenever standard deviation exceeded $30 \mu \mathrm{V}$ within a gliding window of $200 \mathrm{msec}$ or $60 \mu \mathrm{V}$ within a sliding window of 100 msec. ERPs were averaged within conditions for each participant, and the data were analyzed by computing the mean amplitude in a selected latency window (500-700 msec) relative to a 200-msec prestimulus baseline for the $\mathrm{N} 5$ component. The latency window was chosen after a 50-msec step-by-step analysis, indicating that larger significant effects were present between 500 and 700 msec.

For statistical evaluation, ERPs were analyzed by repeated-measures analyses of variance (ANOVAs) for four regions of interest (ROIs): left anterior (mean of F5, F3, FC5, FC3), right anterior (mean of F6, F4, FC6, FC4), left posterior (mean of P5, P3, CP5, CP3), right posterior (mean of $\mathrm{P} 6, \mathrm{P} 4, \mathrm{CP} 6, \mathrm{CP} 4$ ). The same ROIs were used for the components elicited by the deviant timbre sequences, with selected latency windows, relative to a 200-msec prestimulus, from 100 to $200 \mathrm{msec}$ for the MMN, 300-1000 msec for the P300, and 450$1200 \mathrm{msec}$, for the late negative component. ANOVAs were conducted with factors musical expertise (musicians vs. nonmusicians) as a between-subject factor, harmonic function (tonic vs. subdominant), context (with vs. without the subdominant in the context), laterality (left vs. right), and region (anterior vs. posterior) as within-subject factors. 


\section{RESULTS}

\section{Behavioral Data}

Participants detected, on average, 96.85\% ( $S D=4.36)$ of the timbre-deviant chords (nonmusicians: $96.42 \%$; musicians: $97.25 \%$; the difference between groups was not significant, $F>1)$. There were $0.26 \%(S D=1.05)$ false alarms (nonmusicians: 0.38\%; musicians: $0.13 \%$; the difference between groups was not significant). Both groups performed well above chance [nonmusicians: $t(18)=39.56, p<.0001 ;$ musicians: $t(20)=59.78$, $p<.0001$ ] , indicating that both groups easily mastered the task (despite watching the silent movie).

\section{ERP Data}

\section{Harmonic Function (I vs. IV)}

As shown in Figure 2, subdominant compared to tonic chords elicited a larger negative component around 500-700 msec. The difference between subdominant and tonic chords was larger in the anterior region and not lateralized. The effect was clearly observable in musicians, but it failed to reach statistical significance with nonmusicians. With nose reference, this negativity inverted polarity at mastoid electrodes. Moreover, the difference between subdominant and tonic was larger when the subdominant occurred in the previous context (compared to sequences in which the subdominant did not occur in the previous context).

A repeated-measures ANOVA with factors musical expertise (musicians, nonmusicians), context (with, without the subdominant in the context), harmonic function (tonic, subdominant), region (anterior, posterior), and laterality (left, right) was carried out for a time window from 500 to $700 \mathrm{msec}$, and indicated (a) an effect of harmonic function $[F(1,38)=6.52, p=.0148]$, reflecting that the negativity was larger for the less stable subdominant than for the stable tonic chords; (b) an interaction between factors harmonic function and musical expertise $[F(1,38)=4.68, p=.0370]$, reflecting that the effect of harmonic function reached significance only in musicians; (c) an interaction between factors harmonic function and region $[F(1,38)=11.26, p=$ .0018], reflecting that the effect of harmonic function was larger over anterior regions; and (d) an interaction between factors harmonic function and context $[F(1,38)=5.16, p=.0288]$, reflecting that the effect of harmonic function was significant only when the subdominant occurred in the context. There was no interaction between factors harmonic function and laterality.

ANOVAs conducted separately for musicians and nonmusicians indicated for musicians, (a) an effect of harmonic function $[F(1,20)=18.11, p=.0004]$; (b) an interaction between factors harmonic function and region $[F(1,20)=15.43, p=.0008]$, and (c) an interaction

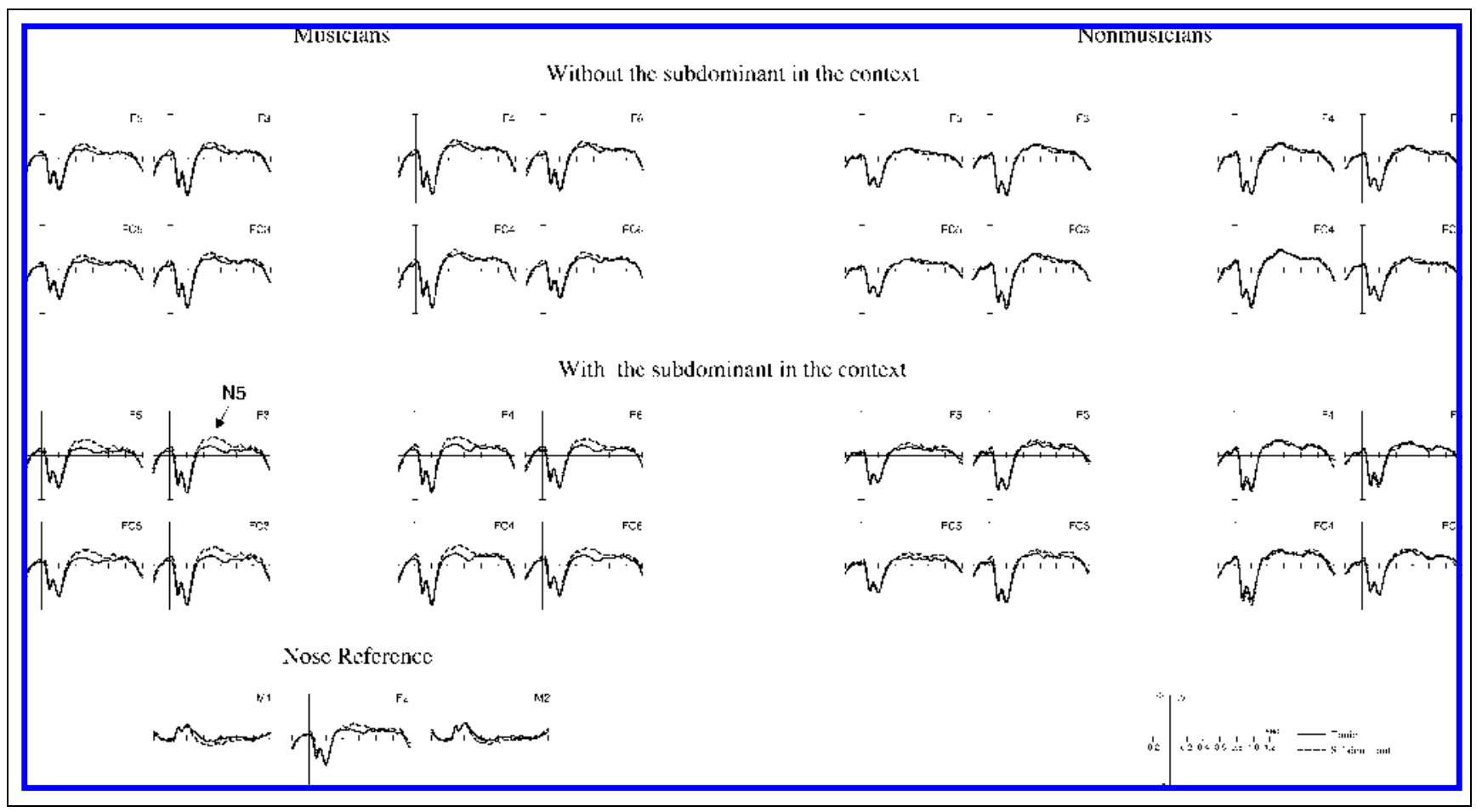

Figure 2. ERPs elicited by chords at the eighth position, separately for musicians (left panel) and nonmusicians (right panel). Subdominant chords elicited a larger N5 than tonic chords (maximal around 500-700 msec), especially in musicians and with the subdominant in the context. Reference is the mean of M1, M2. When a nose reference is used, the N5 inverted polarity at mastoid electrodes for musicians, sequences with the subdominant in the context. 
between factors harmonic function and context $[F(1,20)=$ $5.78, p=.0261]$, indicating that the effect of harmonic function is significant only when the subdominant occurred in the context $[F(1,20)=19.60, p<.001]$ (when the subdominant did not occur in the context, $F<1$ ). For the nonmusicians, no significant effect was observed.

To further investigate (1) whether the N5 effect is similar for tonic and subdominant targets in both with and without the subdominant in the context and (2) whether the N5 component is similar to the N400 component regarding the fact that the amplitude of the N400 component decreases with repetition, additional contrast analyses were performed for the data of musicians (who showed a significant N5 effect). A contrast analysis for the subdominant chords in both with and without the subdominant in the context conditions showed no significant difference, indicating that the N5 effect elicited by subdominant chords in both with and without the subdominant in the context conditions is similar $[F(1,20)=1.61, p=.22]$. In addition, a contrast analysis for the tonic chords in both with and without the subdominant in the context conditions showed that the reduction of the $\mathrm{N} 5$ effect in the condition without the subdominant in the context is mainly due to the fact that the tonic chord elicited a larger N5 effect in the condition without the subdominant in the context compared to the condition with the subdominant in the context $[F(1,20)=5.34, p=.032]$.

Note that when neither the subdominant nor the tonic occurs in the previous context, the sequences mostly comprised minor and diminished chords, which are probably not sufficient to clearly establish the key. To test whether the establishment of the key is more difficult when the subdominant does not appear in the context compared to when the subdominant appears in the context, the key-finding algorithm developed by Krumhansl (1990) was applied to the sequences using the Matlab-implementation of Eerola and Toiviainen (2004). This algorithm returns the correlation of the pitch class distribution of each sequence with the key profiles obtained by Krumhansl and Kessler (1982). The correlations give a measure of the strength of each possible key. On average, the key of the sequences with the subdominant in the context obtained a correlation of .74 with the corresponding key profile, which is significantly higher than the correlation of .62 obtained for the sequences without the subdominant in the context $[t(23)=7.58, p<.001]$.

\section{Timbre MMN}

As shown in Figure 3, timbre deviants elicited an MMN that was largest over central electrodes and inverted polarity with nose reference at mastoid electrodes, although not as clear as usually observed. The MMN was followed by a parietal P300 that peaked around 450-600 msec, and by a late frontal negativity that peaked around 650-750 msec.
A repeated-measures ANOVA with factors deviance (standard, deviant), musical expertise (musicians, nonmusicians), region (anterior, posterior), and laterality (left, right) was carried out for a time window from 100 to $200 \mathrm{msec}(\mathrm{MMN})$, indicating an effect of deviance $[F(1,38)=194.51, p<.0001]$, an interaction between factors deviance and laterality $[F(1,38)=11.15, p=$ .0019], indicating that the MMN was right lateralized with a larger amplitude in the right $(3.69 \mu \mathrm{V})$ than in the left $(3.32 \mu \mathrm{V})$. Additional analyses were performed for the MMN effect on the electrodes $\mathrm{Fz}, \mathrm{Cz}$, and $\mathrm{Pz}$. The MMN effect was significantly larger for $\mathrm{Cz}(5.54 \mu \mathrm{V})$ than $\mathrm{Fz}[3.89 \mu \mathrm{V}, F(1,38)=61.02, p<.001]$ and $\mathrm{Pz}[4.48 \mu \mathrm{V}$, $F(1,38)=28.40, p<.001]$. The MMN effect was significantly larger for $\mathrm{Pz}$ than $\mathrm{Fz}[F(1,38)=5.38, p<$ $.05]$. This analysis suggests a more centro-parietal distribution for the timbre MMN.

An analogous ANOVA for the P300 (time window from 300 to $1000 \mathrm{msec}$ ) indicated an interaction between factors deviance and region $[F(1,38)=217.87, p<$ $.0001]$, reflecting that the P300 was largest over posterior regions $[F(1,38)=61.52, p<.0001]$.

An analogous ANOVA for the frontal late negativity (time window from 450 to $1200 \mathrm{msec}$ ) indicated (a) an interaction between factors deviance and region $[F(1,38)=$ $258.76, p<.0001]$, reflecting that the frontal late negativity was largest over anterior regions $[F(1,38)=80.96$, $p<.0001]$, and (b) an interaction between factors deviance and laterality $[F(1,38)=11.89, p=.001]$.

None of the tests indicated any interaction between factors deviance and musical expertise.

\section{DISCUSSION}

The present study attempted to accommodate contradictory results obtained in previous ERP studies evaluating the processing of musical syntax with chord sequences: Some studies reported that positive deflection reflected the processing of harmonic structure (Regnault et al., 2001; Patel et al., 1998; Janata, 1995), whereas others reported negative deflections (for a review, see Koelsch \& Friederici, 2003).

In the present experiment, we investigated similar irregularities as Regnault et al. (2001) with the experimental procedure used by Koelsch et al. (2000). First of all, contrary to Regnault et al., who investigated the same harmonic irregularities (tonic vs. subdominant), no positive deflection (P300) was observed. In their study, participants were asked to indicate for each target chord if it was consonant or dissonant. In the present study, the target chords were not task-relevant. The absence of the P300 in the present study indicates that the occurrence of the P300 would be related to the task relevance of the target chords. This suggests that the P300 observed by Regnault et al. was presumably reflecting a task-relevance mechanism, which was affected by the processing of harmonic function rather than the 


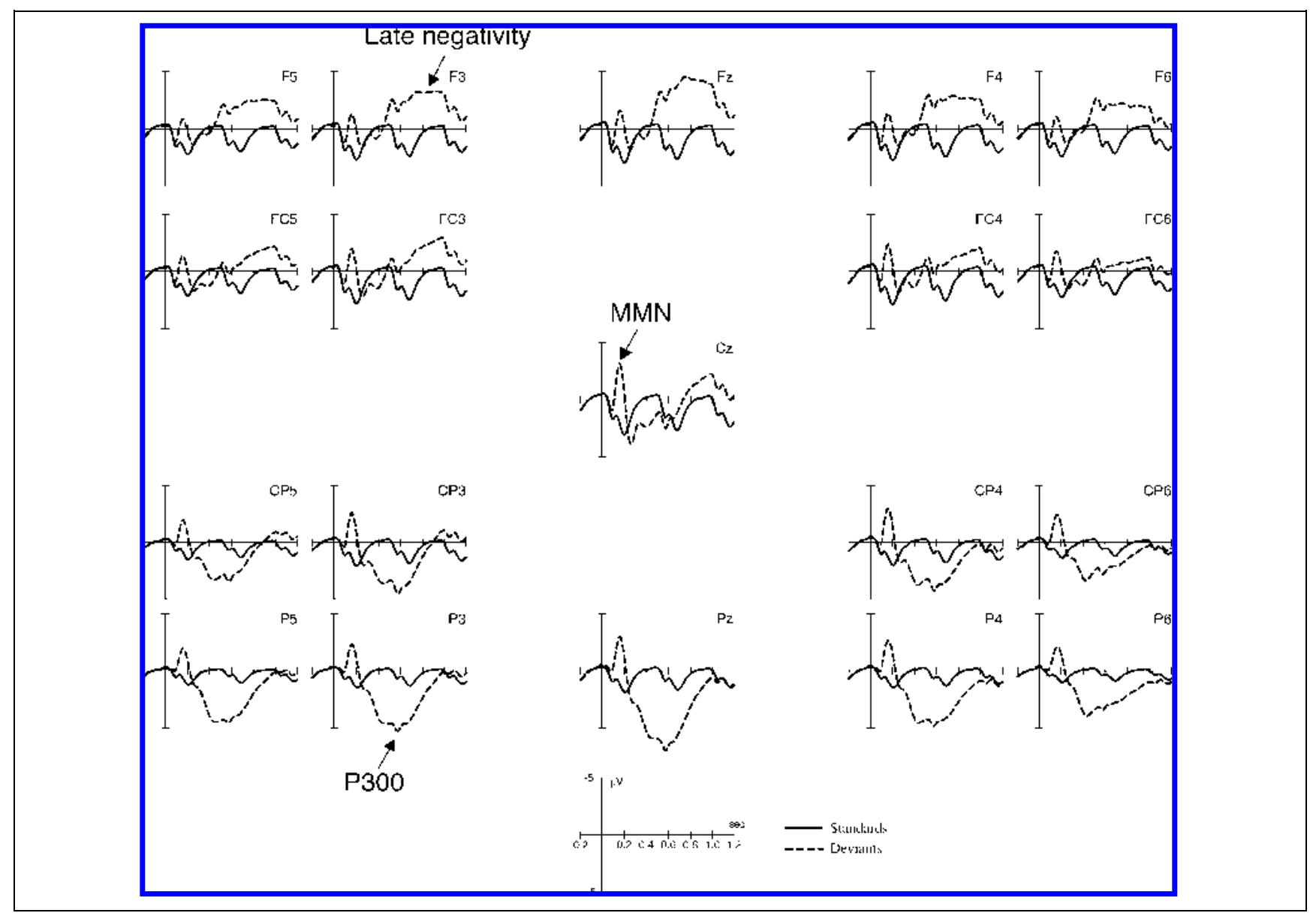

Figure 3. ERPs to standard and timbre-deviant chords (averaged across both groups, musicians and nonmusicians). Deviant chords elicited a larger MMN (maximal around 100-200 msec), a larger P300 (maximal around 450-600 msec), and a larger late negativity (maximal around 650-750 msec) than standard chords. Reference is the mean of M1, M2. None of the ERP effects differed between groups.

processing of harmonic function per se. Furthermore, the elicitation of a large P300 for the timbre-deviant chords, which were task-relevant in the present study, corroborates this hypothesis.

A second main outcome is that the present results partially replicated previous studies reported negative deflections (Koelsch, Schmidt, et al., 2002; Koelsch, Schröger, et al., 2002; Koelsch et al., 2000): The less stable subdominant chords elicited a larger N5 than the stable tonic chords. This $\mathrm{N} 5$ effect is interpreted as reflecting a process of harmonic integration and is reminiscent of the semantic integration process reflected by the N400 originally observed in the language domain (Kutas \& Hillyard, 1980, 1984). The better an element fits with the previous context, the better it can be integrated with the context, and the smaller is the amplitude of the N400. In the case of musical structure, the tonic chord, more stable and more expected than the subdominant chord, fits better with the context. As a consequence, the less stable subdominant chord is more difficult to integrate into the context than the stable tonic chord and leads to an increase in the N5 amplitude. It is worth noting that an inspection of the results of Regnault et al. suggests an N5-like effect to be larger for subdominant chords compared to tonic chords when both were consonant. This N5 effect was not analyzed directly, although there is some evidence for its reliability in the 300-800 msec range, with a more centroparietal distribution.

In addition, the results demonstrated that the musical context (with or without the subdominant) modulated the amplitude of this N5 effect. To disentangle sensory and cognitive processes present in harmonic priming, Bigand et al. (2003) manipulated the occurrence of the less stable subdominant chord in the previous context. In one condition, neither the tonic nor the subdominant chord occurred in the previous context. In a second condition, the subdominant occurred one or two times, whereas the tonic never occurred in the previous context. Independently of both these conditions, the tonic was always processed faster and better than the subdominant chord, suggesting that the performance of the listeners relied more on cognitive than sensory processes. However, in the present study, the difference between tonic and subdominant chords was larger when the subdominant occurred in the previous context than when 
it did not. As in Bigand et al., a sensory effect was ruled out. If the N5 component reacts exactly like the N400, the repetition of a target event should reduce the amplitude of the N5. If sensory effects (repetition of the subdominant in the previous context) make subdominant chords easier to integrate in the context compared to tonic chords, at the extreme, a larger N5 is expected for tonic chords compared to subdominant chords or at least a reduced $\mathrm{N} 5$ for the subdominant chord when it was repeated in the context compared to when it was not. First, the results show that the subdominant chord elicited a larger N5 than the tonic when the subdominant appeared in the previous context, suggesting that even when repeated, the subdominant chord remained more difficult to integrate into the context than the expected tonic chord. Second, additional contrast analyses indicated that the subdominant elicited a similar negative deflection in both conditions with or without the subdominant in the context, suggesting that the amplitude of the N5, for the subdominant chord, did not decrease with repetition. This result is in accordance with recent results of Bigand, Tillmann, Poulin-Charronnat, and Manderlier (2005), who did not find an advantage of repetition priming with chords. When using pairs of chords, no significant difference between chord repetition and harmonic relatedness or facilitated processing for harmonically related targets was observed. When using chord sequences, harmonic priming was shown to depend more on the harmonic function of the target in the preceding context than on target repetition. These findings suggest that repetition in music is different than repetition in other domains and, as consequence, it is likely that the $\mathrm{N} 5$ does not react exactly like the N400. The absence of difference between tonic and subdominant chords when the subdominant did not occur in the previous context might rely on the absence of these two hierarchically stable chords in the context. When neither the subdominant nor the tonic occurs in the previous context, the sequences mostly comprised minor and diminished chords, which make the key more difficult to be established. This argument is supported by the finding that the key of the sequences with the subdominant in the context correlates higher with the corresponding Krumhansl and Kessler's key profile than the key of the sequences without the subdominant in the context. Consequently, the key of the sequences in which the subdominant does not occur in the context is less clearly established. Because the perception of harmonic function depends on the perception of the key, the difference between tonic and subdominant is more difficult to perceive when the subdominant does not appear in the previous context presumably leading to a reduction of the $\mathrm{N} 5$ effect.

Contrast analyses performed on tonic and subdominant chords supported this hypothesis and indicated that the tonic chord is the most disadvantaged target chord. The tonic target chord elicited a larger N5 when the subdominant did not occur in the previous context than when the subdominant occurred in the previous context, whereas the N5 effect is similar for the subdominant target chords in both conditions. If the inference of the key is more difficult when neither the tonic nor the subdominant chord occurred in the context and as the formation of musical expectancy strongly depend on the inference of the key, it is more difficult to form an expectancy on the tonic chord, making it more difficult to integrate into the context. The subdominant target chord, in both with and without the subdominant in context, does not correspond to the expectancy formed by the listeners and is similarly more difficult to integrate with the previous context in both conditions, showing no difference in the amplitude of the N5 effect.

Although our data elicited an N5 effect, they also differed from the results of studies from Koelsch, Schmidt, et al. (2002), Koelsch, Schröger, et al. (2002), and Koelsch et al. (2000): No ERAN was elicited. Although the presence of an N5 demonstrates that this component can be elicited by purely cognitive factors (because the present harmonic manipulations did not confound with sensory deviance), the absence of the ERAN in the present study might lead to the assumption that the ERAN elicited in some previous studies was mainly due to sensory factors. However, recent data showed that the ERAN can be elicited by harmonically irregular chords which do not represent sensory deviants (Koelsch, 2005), and preliminary data from our lab (Poulin-Charronnat, Bigand, \& Koelsch, 2005) suggest that early anterior negativities are also elicited by the sequences used in the present study when the target chords are task-relevant. In any case, the present data strongly suggest that no ERAN is elicited by very subtle harmonic irregularities under a condition in which the musical stimuli are ignored by listeners. Another possible way to account for the absence of ERAN would be to consider the difference in the syntactic irregularities evaluated. The ERAN, initially taken as a sound expectancy violation, might reflect the perceptive detection of the violation and might be elicited only by stronger harmonic irregularities. It is likely that the harmonic violations used in the studies from Koelsch et al. (2000) (tonic vs. Neapolitan) differ in nature from the ones used in the present study (tonic vs. subdominant) because the irregular Neapolitan chords did not belong to the key context, whereas the subdominant chord did. As a consequence, the subdominant irregularities are perceived as less salient than the Neapolitan ones and might not be salient enough to elicit an ERAN.

Thus, the absence of ERAN might be due to the fact that the subdominant chord is a less salient irregularity than a Neapolitan chord. To further evaluate this issue, we conducted an additional behavioral experiment. In Koelsch et al. (2000), 97\% of nonmusicians detected the Neapolitan as an irregular chord at the end of fivechord sequences. For comparison with Koelsch et al., 26 
nonmusicians were presented with the eight-chord sequences and were required to indicate as fast as possible if the last chord was a regular chord (a chord on which the sequence ended well, tonic) or an irregular chord (a chord on which the sequence ended not so well, subdominant). The tonic chords (855.87 msec, $71.77 \%$ of correct choices) were processed faster and more accurately than the subdominant chords (926.17 $\mathrm{msec}$, $53.85 \%$ of correct choices). This difference was significant for both percent of correct choices and response times [respectively $t(25)=4.58, p<.001$ and $t(25)=$ $-3.80, p<.001]$ and the percentage of correct choices was above chance for the tonic targets $[t(25)=8.44, p<$ $.0001]$, but not for the subdominant targets $[t(25)=$ $1.13, p>.10]$. The low level of correct choices for the subdominant chords, indicating that this chord is not perceived as such a bad ending of the sequence, demonstrates that the difference between tonic and subdominant is less salient compared, for example, to the Neapolitan chords used in Koelsch et al. (2000). This finding suggests that, under preattentive conditions, the ERAN may be elicited only by more salient harmonic irregularities and not by very subtle ones.

Finally, a third outcome indicates that the difference between tonic and subdominant may be processed preattentively only by musically expert listeners. This extends previous ERP results reporting preattentive processing of strong harmonic irregularities in nonmusicians (Koelsch, Schröger, et al., 2002), in that nonmusicians were unable to preattentively process less salient harmonic irregularities. However, it cannot be ruled out that some participants (especially nonmusicians) may have done the very easy timbre task in the mental background while focusing on the movie, whereas others (especially musicians) may have focused on the music and paid much less attention to the movie. This difference in attentional focus might theoretically also account for the absence of an N5 in nonmusicians. However, the behavioral data argue against this possibility: If musicians were paying more attention to the musical sequences, their performance in the very easy timbre detection task should have been nearly perfect (around 99\% of correct detections) and better than those of nonmusicians, which was not the case. In addition, it is important to note that participants listened to the same set of monotonous chord sequences over and over again for 2.5 hours. Thus, it is very probable that all participants found the movie much more attractive. For these reasons, it is highly likely that the subtle harmonic irregularities were processed preattentively by musicians, but not by nonmusicians (leading to an $\mathrm{N} 5$ effect in musicians, but not in nonmusicians). In addition, concerning the processing of timbre deviance, both musicians and nonmusicians elicited the same three components, an MMN, a P300, and a late negativity. These ERP effects did not differ between groups, indicating that both musicians and nonmusi- cians process this violation in harmonic spectra in a preattentive way as already demonstrated in previous research (Tervaniemi et al., 1997). Nevertheless, future studies could first investigate if the difference between musicians and nonmusicians, as well as the N5 effect observed in musicians, can also be observed under more controlled and more demanding conditions of attentional distraction (such as the performance of a visual tracking task during the presentation of the chord sequences). Second, future studies should address whether the subdominant chords elicit a significant response, especially in nonmusicians, under attentive conditions in which the harmonic function of the final target chord is not task-relevant and maybe by using a more naturalistic task than a detection of timbre deviants.

\section{Acknowledgments}

This research was supported by a grant awarded to Bénédicte Poulin-Charronnat from the Fyssen Foundation.

We thank Katrin Bittrich and Sibylle Herholz for their assistance in data collection.

Reprint requests should be sent to Bénédicte Poulin-Charronnat, Max-Planck-Institute for Human Cognitive and Brain Sciences, Stephanstraße 1A, 04103 Leipzig, Germany, or via e-mail: poulin@ cbs.mpg.de.

\section{REFERENCES}

Bharucha, J., \& Krumhansl, C. L. (1983). The representation of harmonic structure in music: Hierarchies of stability as a function of context. Cognition, 13, 63-102.

Bigand, E., Poulin, B., Tillmann, B., Madurell, F., \& D’Adamo, D. A. (2003). Sensory versus cognitive components in harmonic priming. Journal of Experimental Psychology: Human Perception and Performance, 29, 159-171.

Bigand, E., \& Poulin-Charronnat, B. (in press). Are we "experienced listeners"? A review of the musical capabilities that do not depend on formal musical training. Cognition.

Bigand, E., Tillmann, B., Poulin, B., D'Adamo, D. A., \& Madurell, F. (2001). The effect of harmonic context on phoneme monitoring in vocal music. Cognition, 81 , B11-B20.

Bigand, E., Tillmann, B., Poulin-Charronnat, B., \& Manderlier, D. (2005). Repetition priming: Is music special? Quarterly Journal of Experimental Psychology, 58A, 1347-1375.

Eerola, T., \& Toiviainen, P. (2004). MIDI Toolbox: MATLAB Tools for Music Research. University of Jyväskylä: Kopijyvä, Jyväskylä, Finland. Retrieved from www.jyu.fi/ musica/miditoolbox/ in May 2004.

Janata, P. (1995). ERP measures assay the degree of expectancy violation of harmonic contexts in music. Journal of Cognitive Neuroscience, 7, 153-164.

Koelsch, S. (2005). Neural substrates of processing syntax and semantics in music. Current Opinion in Neurobiology 15, 207-212.

Koelsch, S., \& Friederici, A. D. (2003). Toward the neural basis of processing structure in music. Comparative results of different neurophysiological investigation methods. Annals of the New York Academy of Sciences. 999, 15-28. 
Koelsch, S., Gunter, T., Friederici, A. D., \& Schröger, E. (2000). Brain indices of music processing: "Nonmusicians" are musical. Journal of Cognitive Neuroscience, 12 , 520-541.

Koelsch, S., Schmidt, B.-H., \& Kansok, J. (2002). Effects of musical expertise on the early right anterior negativity: An event-related brain potential study. Psychopbysiology 39, 657-663.

Koelsch, S., Schröger, E., \& Gunter, T. C. (2002). Music matters: Preattentive musicality of the human brain. Psychophysiology, 39, 38-48.

Krumhansl, C. L. (1990). Cognitive foundations of musical pitch. New York: Oxford University Press.

Krumhansl, C. L., \& Kessler, E. J. (1982). Tracing the dynamic changes in perceived tonal organization in a spatial representation of musical keys. Psvchological Review, 89, 334-368.

Kutas, M., \& Hillyard, S. A. (1980). Reading senseless sentences: Brain potentials reflect semantic incongruity. Science, 207, 203-205.

Kutas, M., \& Hillyard, S. A. (1984). Brain potentials during reading reflect word expectancy and semantic association. Nature, 307, 161-163.
Patel, A. D., Gibson, E., Ratner, J., Besson, M., \& Holcomb, P. J. (1998). Processing syntactic relations in language and music: An event-related potential study. Journal of Cognitive Neuroscience, 10, 717-733.

Poulin-Charronnat, B., Bigand, E., \& Koelsch, S. (2005). Attention effects on the processing of musical syntax in nonmusicians. Poster presented at the Neurosciences and Music: II. From perception to performance conference, May 5-8, The Westin, Leipzig, Germany.

Poulin-Charronnat, B., Bigand, E., Madurell, F., \& Peereman, R. (2005). Musical structure modulates semantic priming in vocal music. Cognition, 94, B67-B78.

Regnault, P., Bigand, E., \& Besson, M. (2001). Different brain mechanisms mediate sensitivity to sensory consonance and harmonic context: Evidence from auditory event-related brain potentials. Journal of Cognitive Neuroscience, 13, 241-255.

Tervaniemi, M., Winkler, I., \& Näätänen, R. (1997). Pre-attentive categorization of sounds timbre as revealed by event-related potentials. NeuroReport, 8, 2571-2574.

Tillmann, B., Bharucha, J. J., \& Bigand, E. (2000). Implicit learning of tonality: A self-organizing approach. Psvchological Review, 107, 885-913. 